\title{
REAL EIGENVALUES OF A NON-SELF-ADJOINT PERTURBATION OF THE SELF-ADJOINT ZAKHAROV-SHABAT OPERATOR
}

\author{
K. HIROTA
}

\begin{abstract}
We study the eigenvalues of the self-adjoint Zakharov-Shabat operator corresponding to the defocusing nonlinear Schrödinger equation in the inverse scattering method. Real eigenvalues exist when the square of the potential has a simple well. We derive two types of quantization condition for the eigenvalues by using the exact WKB method, and show that the eigenvalues stay real for a sufficiently small non-selfadjoint perturbation when the potential has some $\mathcal{P} \mathcal{T}$-like symmetry.
\end{abstract}

\section{INTRODUCTION}

We consider the eigenvalue problem

$$
L \boldsymbol{u}(x)=\lambda \boldsymbol{u}(x),
$$

for the first order $2 \times 2$ differential system on the line:

$$
L:=\left(\begin{array}{cc}
i h \frac{d}{d x} & -i A(x) \\
i A(x) & -i h \frac{d}{d x}
\end{array}\right),
$$

where $h$ is a small positive parameter, $\lambda$ is a spectral parameter, $\boldsymbol{u}(x)$ is a column vector, and $A(x)$ is a real-valued potential. This operator is called the Zakharov-Shabat operator, which is one of the two operators in the Lax pair for the defocusing nonlinear Schrödinger equation:

$$
i h \frac{\partial \psi}{\partial t}+\frac{h^{2}}{2} \frac{\partial^{2} \psi}{\partial x^{2}}-|\psi|^{2} \psi=0, \quad \psi=\psi(t, x),
$$

and the scattering theory of $L$ plays an important role in the analysis of the solutions of the initial value problem for this equation.

The operator $L$ is self-adjoint, and it is expected that $L$ has real eigenvalues when $A(x)^{2}$ has a well. In the first part of our study, we derive the BohrSommerfeld type quantization condition for the eigenvalues of $L$ under the following assumption.

Assumption (A1). Let $A(x)$ be a real-valued function analytic in $D:=$ $\{z \in \mathbb{C} ;|\operatorname{Imz}|<\delta\}$ for some $\delta>0$, and $\lambda_{0}$ a positive real number satisfying the following conditions:

Keywords: Zakharov-Shabat eigenvalue problem, exact WKB method, quantization condition. 
K. HIROTA

(1) There exist two real numbers, $\alpha_{0}$ and $\beta_{0}\left(\alpha_{0}<\beta_{0}\right)$ such that $|A(x)|=$ $\lambda_{0}, x \in \mathbb{R}$ if and only if $x=\alpha_{0}, \beta_{0}$.

(2) $A^{\prime}\left(\alpha_{0}\right) A^{\prime}\left(\beta_{0}\right) \neq 0$.

(3) $|A(x)|<\lambda_{0}$ for $\alpha_{0}<x<\beta_{0}$, and $|A(x)|>\lambda_{0}$ for $x<\alpha_{0}$ and $x>\beta_{0}$.

(4) $\liminf _{|x| \rightarrow \infty}|A(x)|>\lambda_{0}$.

This assumption permits two types of potentials. One is a simple well type where $A\left(\alpha_{0}\right)=A\left(\beta_{0}\right)$, and the other is monotonic type where $A\left(\alpha_{0}\right)=$ $-A\left(\beta_{0}\right)$. In both cases, $A(x)^{2}$ has a simple well, see Figure 1 .
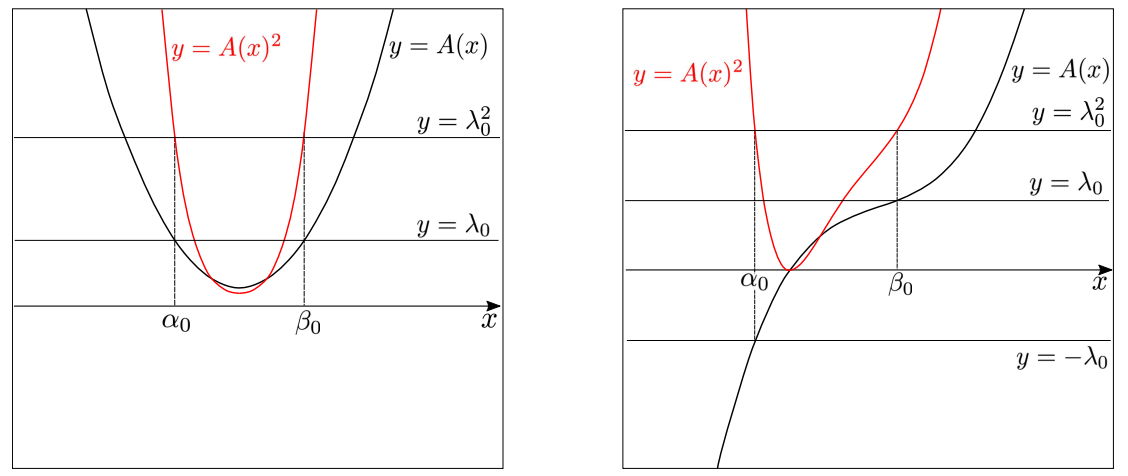

FiguRE 1. Examples of the potential $A(x)$.

For $\lambda \in \mathbb{R}$ close enough to $\lambda_{0}$, the function $\lambda^{2}-A(x)^{2}$ has exactly two real zeros $\alpha(\lambda)$ and $\beta(\lambda)$, close to $\alpha_{0}$ and $\beta_{0}$ respectively, and we define the action integral

$$
I(\lambda):=\int_{\alpha(\lambda)}^{\beta(\lambda)} \sqrt{\lambda^{2}-A(t)^{2}} d t .
$$

Then, we obtain the following quantization conditions.

Theorem 1.1. Assume (A1). In the case $A\left(\alpha_{0}\right)=A\left(\beta_{0}\right)$, there exist positive constants $\delta$ and $h_{0}$, and a function $r_{+}(\lambda, h)$ bounded on $\left[\lambda_{0}-\delta, \lambda_{0}+\right.$ $\delta] \times\left(0, h_{0}\right]$ such that $\lambda \in\left[\lambda_{0}-\delta, \lambda_{0}+\delta\right]$ is an eigenvalue of $L$ for $h \in\left(0, h_{0}\right]$ if and only if

$$
I(\lambda)=\left(k+\frac{1}{2}\right) \pi h+h^{2} r_{+}(\lambda, h)
$$

holds for some integer $k$. In the case $A\left(\alpha_{0}\right)=-A\left(\beta_{0}\right)$, there exist positive constants $\delta$ and $h_{0}$, and a function $r_{-}(\lambda, h)$ bounded on $\left[\lambda_{0}-\delta, \lambda_{0}+\delta\right] \times\left(0, h_{0}\right]$ such that $\lambda \in\left[\lambda_{0}-\delta, \lambda_{0}+\delta\right]$ is an eigenvalue of $L$ for $h \in\left(0, h_{0}\right]$ if and only if

$$
I(\lambda)=k \pi h+h^{2} r_{-}(\lambda, h)
$$

holds for some integer $k$.

Next, we add a small complex perturbation to the potential $A(x)$ :

$$
A_{\varepsilon}(x)=A(x)+i \varepsilon B(x)
$$


with a real-valued function $B(x)$ and a positive small parameter $\varepsilon$, and consider the eigenvalues of $L_{\varepsilon}$

$$
L_{\varepsilon}:=\left(\begin{array}{cc}
i h \frac{d}{d x} & -i A_{\varepsilon}(x) \\
i A_{\varepsilon}(x) & -i h \frac{d}{d x}
\end{array}\right) .
$$

This operator is no longer self-adjoint, and eigenvalues become complex in general.

In the case of Schrödinger operator, $\mathcal{P} \mathcal{T}$-symmetry has been expected to be an alternative to the self-adjointness in order to have real eigenvalues. In recent studies, Boussekkine and Mecherout considered in for the Schrödinger operator with $\mathcal{P} \mathcal{T}$-symmetry

$$
P_{\varepsilon}:=-h^{2} \frac{d^{2}}{d x^{2}}+V(x)+i \varepsilon W(x),
$$

where $V(x)$ is a simple well even function and $W(x)$ is an odd function, and showed that reality of eigenvalues also holds for sufficiently small $\varepsilon$ and $h$. After that, Boussekkine, Mecherout, Ramond and Sjöstrand studied in [8] the double well case with $\mathcal{P} \mathcal{T}$-symmetry, and found that the eigenvalues stay real only for exponentially small $\varepsilon$ with respect to $h$.

In this paper, we continue in this direction and prove that a sufficiently small complex perturbation of the self-adjoint Zakharov-Shabat operator $L_{\varepsilon}$ has real eigenvalues when $A(x)$ and $B(x)$ have some $\mathcal{P} \mathcal{T}$-like symmetry symmetry in the case where $A(x)^{2}$ has a simple well, even though the perturbed operator $L_{\varepsilon}$ is non-self-adjoint. Recalling that the condition where $P_{\varepsilon}$ is $\mathcal{P} \mathcal{T}$-symmetric is equivalent to one where $V(x)$ is an even function and $W(x)$ is an odd function (see [4] or [8]), we assume the following symmetry properties for $A(x)$ and $B(x)$.

Assumption (A2). Let $B(x)$ be real-valued, analytic and bounded on $\mathbb{R}$. $A(x)$ and $B(x)$ satisfy for $x \in \mathbb{R}$ either

$$
A(x)=A(-x), \quad B(x)=-B(-x),
$$

or

$$
A(x)=-A(-x), \quad B(x)=B(-x)
$$

The following theorem shows that the eigenvalues of $L_{\varepsilon}$ are real for sufficiently small $\varepsilon$ and $h$.

Theorem 1.2. Assume (A1) and (A2). Then there exist positive constants $\varepsilon_{0}$ and $h_{0}$ such that $\sigma\left(L_{\varepsilon}\right) \cap\left\{\lambda \in \mathbb{C}:\left|\lambda-\lambda_{0}\right|<\varepsilon_{0}\right\} \subset \mathbb{R}$ when $0<\varepsilon \leq \varepsilon_{0}$ and $0<h \leq h_{0}$.

To prove Theorem 1.1 and 1.2 , we use the exact WKB method. In Section 2, we mention the exact WKB solutions for (1.1) and introduce three important properties. These exact WKB solutions are used in Section 3 to derive the quantization conditions (1.3) and (1.4). After that, we consider the perturbed case, and give the proof for Theorem 1.2 in Section 4. 


\section{ExACT WKB SOLUTiOns}

We construct solutions to (1.1) by the exact WKB method. This method was proposed by Gérard and Grigis in [7], and extended to $2 \times 2$ systems by Fujiié, Lasser and Nédélec in [5].

Before the construction, we assume that $\Omega$ is a simply connected open subset of $D$, where $A(x)^{2}-\lambda^{2}$ does not vanish. Following [5], we can construct exact WKB solutions for (1.1) in the form

$$
\boldsymbol{u}^{ \pm}\left(x, h ; \gamma, x_{0}\right)=\left(\begin{array}{cc}
1 & 1 \\
-i & i
\end{array}\right) e^{ \pm z(x ; \gamma) / h} Q(x)\left(\begin{array}{ll}
0 & 1 \\
1 & 0
\end{array}\right)^{\frac{1 \pm 1}{2}} \boldsymbol{w}^{ \pm}\left(x, h ; x_{0}\right)
$$

with base points $\gamma \in D$ and $x_{0} \in \Omega$. Here, $z(x ; \gamma)$ is a phase function

$$
z(x ; \gamma):=\int_{\gamma}^{x} \sqrt{A(t)^{2}-\lambda^{2}} d t
$$

$Q(x)$ is a $2 \times 2$ matrix function

$$
Q(x)=\left(\begin{array}{cc}
H(x)^{-1} & H(x)^{-1} \\
i H(x) & -i H(x)
\end{array}\right), \quad H(x)=\left(\frac{A(x)+\lambda}{A(x)-\lambda}\right)^{\frac{1}{4}},
$$

and $\boldsymbol{w}^{ \pm}\left(x, h ; x_{0}\right)$ are the series

$$
\boldsymbol{w}^{ \pm}\left(x, h ; x_{0}\right)=\left(\begin{array}{c}
w_{\text {even }}^{ \pm}\left(x, h ; x_{0}\right) \\
w_{\text {odd }}^{ \pm}\left(x, h ; x_{0}\right)
\end{array}\right):=\sum_{n=0}^{\infty}\left(\begin{array}{c}
w_{2 n}^{ \pm}(x, h) \\
w_{2 n-1}^{ \pm}(x, h)
\end{array}\right)
$$

constructed by the recurrence equations

$$
\left\{\begin{array}{l}
w_{-1}^{ \pm}=0, w_{0}^{ \pm}=1, \\
\frac{d}{d x} w_{2 n}^{ \pm}=c(x) w_{2 n-1}^{ \pm}, \quad c(x)=\frac{H^{\prime}(x)}{H(x)} \\
\left(\frac{d}{d x} \pm \frac{2 z^{\prime}}{h}\right) w_{2 n-1}^{ \pm}=c(x) w_{2 n-2}^{ \pm},
\end{array}\right.
$$

and the initial conditions

$$
\left.w_{n}^{ \pm}\right|_{x=x_{0}}=0 \quad(n \geq 1) .
$$

These solutions constructed above formally satisfy (1.1). We recall here the following three propositions. The proofs are found in [5] or [7]. The first is about the convergence of series.

Proposition 2.1. Two series $w_{\text {even }}^{ \pm}\left(x, h ; x_{0}\right)$ and $w_{\text {odd }}^{ \pm}\left(x, h ; x_{0}\right)$ are absolutely convergent in a neighborhood of $x_{0}$. Furthermore, $w_{\text {even }}^{ \pm}\left(x, h ; x_{0}\right)$ and $w_{\text {odd }}^{ \pm}\left(x, h ; x_{0}\right)$ are analytic functions in $\Omega$.

The second property is about the Wronskian for two different types of exact WKB solutions.

Proposition 2.2. Let $\gamma, x_{0}, x_{1} \in \Omega$ be the base points. Then, the exact WKB solutions $\boldsymbol{u}^{ \pm}\left(x, h ; \gamma, x_{0}\right)$ and $\boldsymbol{u}^{ \pm}\left(x, h ; \gamma, x_{1}\right)$ satisfy

$$
\mathcal{W}\left(\boldsymbol{u}^{ \pm}\left(x, h ; \gamma, x_{0}\right), \boldsymbol{u}^{\mp}\left(x, h ; \gamma, x_{1}\right)\right)=\mp 4 w_{\text {even }}^{ \pm}\left(x_{1}, h ; x_{0}\right),
$$

where $\mathcal{W}(\boldsymbol{f}, \boldsymbol{g}):=\operatorname{det}(\boldsymbol{f}, \boldsymbol{g})$. This is called the Wronskian formula. 
The final proposition is about the asymptotic property of the exact WKB solution. Let $x_{0} \in \Omega$ be fixed.

Definition 2.3. We denote by $\Omega_{ \pm}$the subset of all $x \in \Omega$ such that there exists a path in $\Omega$ from $x_{0}$ to $x$ along which $\pm \operatorname{Re} z\left(x ; x_{0}\right)$ is strictly increasing.

Theorem 2.4. The functions $w_{\text {even }}^{ \pm}\left(x, h ; x_{0}\right)$ and $w_{\text {odd }}^{ \pm}\left(x, h ; x_{0}\right)$ have the asymptotic expansions as $h \rightarrow 0$ :

$$
\begin{array}{r}
w_{\text {even }}^{ \pm}\left(x, h ; x_{0}\right)-\sum_{n=0}^{N} w_{2 n}^{ \pm}\left(x, h ; x_{0}\right)=\mathcal{O}\left(h^{N+1}\right), \\
w_{\text {odd }}^{ \pm}\left(x, h ; x_{0}\right)-\sum_{n=0}^{N} w_{2 n-1}^{ \pm}\left(x, h ; x_{0}\right)=\mathcal{O}\left(h^{N+1}\right),
\end{array}
$$

in all compact subsets of $\Omega_{ \pm}$.

To find the domain $\Omega_{ \pm}$, we usually consider the Stokes lines, which are level curves of the real part of $z(x ; \gamma)$. In particular, the Stokes lines passing through the point $\gamma_{0} \in D$ are defined as the set

$$
\left\{x \in D ; \operatorname{Re} z\left(x ; \gamma_{0}\right)=\operatorname{Re} \int_{\gamma_{0}}^{x} \sqrt{A(t)^{2}-\lambda^{2}} d t=0\right\} .
$$

Along a path which intersects transversally with the Stokes lines, $\operatorname{Re} z(x)$ or $-\operatorname{Re} z(x)$ is strictly increasing.

\section{Quantization CONDition for the eigenvalues of $L$}

Here we find the quantization condition under Assumption (A1). This is derived from the connection problem of the solutions near the points $\alpha(\lambda)$ and $\beta(\lambda)$ which are zeros of $A(x)^{2}-\lambda^{2}$.

Now, we choose $\alpha(\lambda)$ and $\beta(\lambda)$ for base points of the phase function $z(x)$, and consider the Stokes lines which pass through $\alpha(\lambda)$ and $\beta(\lambda)$. By a simple calculation, we see that the Stokes lines emanate from $\alpha$ at angles of $0,2 \pi / 3$ and $4 \pi / 3$, and emanate from $\beta$ at angles of $\pi / 3, \pi$ and $5 \pi / 3$.

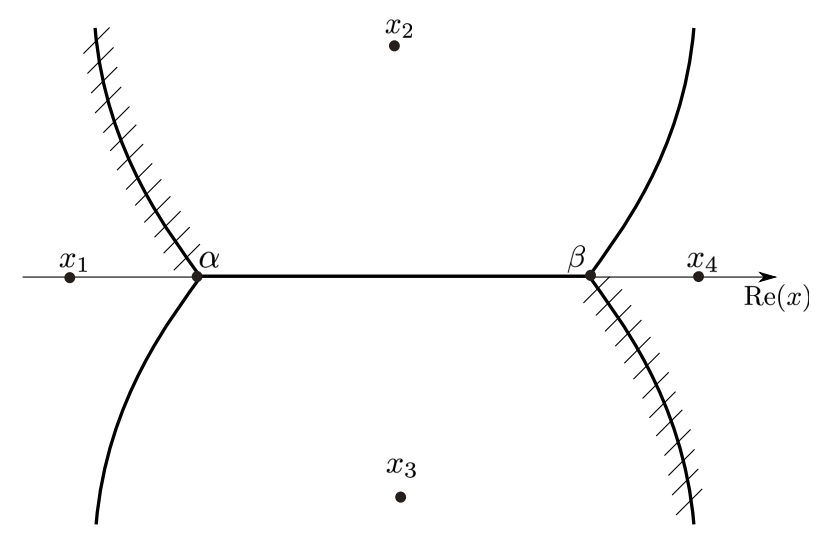

Figure 2. The Stokes lines and base points 
Those Stokes lines separate the complex plane into four sectors as in Figure 2. As $\sqrt{A(x)^{2}-\lambda^{2}}$ and $H(x)$ are multi-valued functions on the complex plane with singularities at $\alpha$ and $\beta$, we set branch cuts emanating at an angle of $2 \pi / 3$ from $\alpha$ and an angle of $5 \pi / 3$ from $\beta$ respectively. We choose the branches such that $\operatorname{Re} \sqrt{A(x)^{2}-\lambda^{2}}$ and $H(x)$ are positive on a part of the real axis $\operatorname{Re}(x)>\beta$.

We take base points for $\boldsymbol{w}^{ \pm}(x, h)$ in each sector as in Figure 2, and define the exact WKB solutions:

$$
\left\{\begin{array}{l}
\boldsymbol{u}_{1}=\boldsymbol{u}^{+}\left(x, h ; \alpha, x_{1}\right) \\
\boldsymbol{u}_{2}=\boldsymbol{u}^{+}\left(x, h ; \alpha, x_{2}\right), \widetilde{\boldsymbol{u}}_{2}=\boldsymbol{u}^{+}\left(x, h ; \beta, x_{2}\right), \\
\boldsymbol{u}_{3}=\boldsymbol{u}^{-}\left(x, h ; \alpha, x_{3}\right), \widetilde{\boldsymbol{u}}_{3}=\boldsymbol{u}^{-}\left(x, h ; \beta, x_{3}\right), \\
\boldsymbol{u}_{4}=\boldsymbol{u}^{-}\left(x, h ; \beta, x_{4}\right) .
\end{array}\right.
$$

Then, we represent $\boldsymbol{u}_{1}$ as a linear combination of $\boldsymbol{u}_{2}$ and $\boldsymbol{u}_{3}$ :

$$
\boldsymbol{u}_{1}=c_{2} \boldsymbol{u}_{2}+c_{3} \boldsymbol{u}_{3},
$$

and $\boldsymbol{u}_{4}$ as

$$
\boldsymbol{u}_{4}=\widetilde{c}_{2} \widetilde{\boldsymbol{u}}_{2}+\widetilde{c}_{3} \widetilde{\boldsymbol{u}}_{3},
$$

where each coefficient depends on $h$ and $\lambda$. We calculate those coefficients by using Theorems 2.2 and 2.4 and obtain the following.

Lemma 3.1. Assume (A1). In the two cases $A(\alpha)= \pm A(\beta)$, the connection coefficients $c_{i}$ and $\widetilde{c_{j}}(i, j \in\{2,3\})$ satisfy

$$
c_{2} \widetilde{c}_{3}=1+\mathcal{O}(h), \widetilde{c}_{2} c_{3}=\mp 1+\mathcal{O}(h) .
$$

as $h \rightarrow 0$.

Proof. Each coefficient is represented in terms of the Wronskians as

$$
\begin{aligned}
& c_{2}=\frac{\mathcal{W}\left(\boldsymbol{u}_{1}, \boldsymbol{u}_{3}\right)}{\mathcal{W}\left(\boldsymbol{u}_{2}, \boldsymbol{u}_{3}\right)}, c_{3}=\frac{\mathcal{W}\left(\boldsymbol{u}_{1}, \boldsymbol{u}_{2}\right)}{\mathcal{W}\left(\boldsymbol{u}_{3}, \boldsymbol{u}_{2}\right)}, \\
& \widetilde{c}_{2}=\frac{\mathcal{W}\left(\boldsymbol{u}_{4}, \widetilde{\boldsymbol{u}}_{3}\right)}{\mathcal{W}\left(\widetilde{\boldsymbol{u}}_{2}, \widetilde{\boldsymbol{u}}_{3}\right)}, \widetilde{c}_{3}=\frac{\mathcal{W}\left(\boldsymbol{u}_{4}, \widetilde{\boldsymbol{u}}_{2}\right)}{\mathcal{W}\left(\widetilde{\boldsymbol{u}}_{3}, \widetilde{\boldsymbol{u}}_{2}\right)} .
\end{aligned}
$$

For $c_{2}$ and $\widetilde{c_{3}}$, we see that

$$
c_{2}=\frac{w_{\text {even }}^{+}\left(x_{3}, h ; x_{1}\right)}{w_{\text {even }}^{+}\left(x_{3}, h ; x_{2}\right)}, \widetilde{c_{3}}=\frac{w_{\text {even }}^{+}\left(x_{4}, h ; x_{2}\right)}{w_{\text {even }}^{+}\left(x_{3}, h ; x_{2}\right)} .
$$

by Theorem 2.2,

Let $\Gamma\left(x_{i}, x_{j}\right)$ denote a path from $x_{i}$ to $x_{j}$. We take $\Gamma\left(x_{1}, x_{3}\right), \Gamma\left(x_{2}, x_{3}\right)$ and $\Gamma\left(x_{2}, x_{4}\right)$, and then notice that they intersect the Stokes lines, see Figure 3. Moreover, $\operatorname{Re} z(x, \cdot)$ increases as $\operatorname{Re}(x)$ increases, or $\operatorname{Im}(x)$ decreases. Therefore, $\operatorname{Re} z(x, \cdot)$ is strictly increasing along those paths.

According to Theorem 2.4, we obtain

$$
\begin{aligned}
& w_{\text {even }}^{+}\left(x_{3}, h ; x_{1}\right)=1+\mathcal{O}(h), w_{\text {even }}^{+}\left(x_{3}, h ; x_{2}\right)=1+\mathcal{O}(h), \\
& w_{\text {even }}^{+}\left(x_{4}, h ; x_{2}\right)=1+\mathcal{O}(h)
\end{aligned}
$$




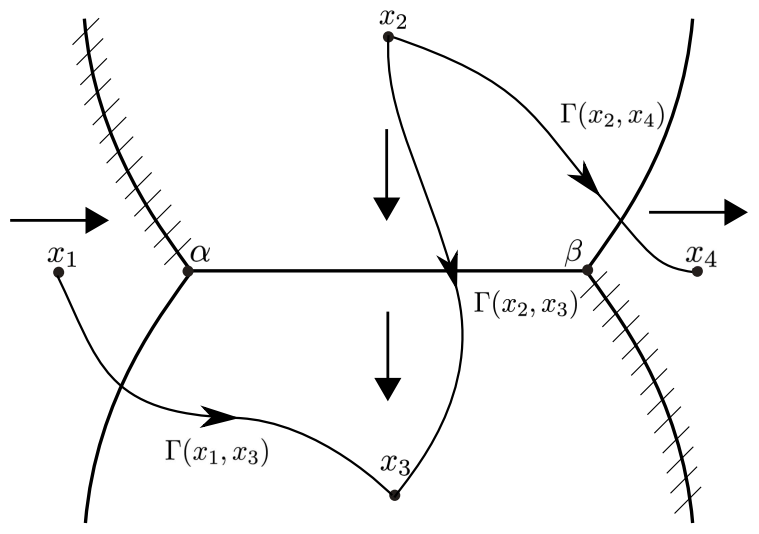

Figure 3. Examples of $\Gamma\left(x_{i}, x_{j}\right)$. Arrows indicate directions along which $\operatorname{Re} z(x)$ increases.

as $h \rightarrow 0$, and we see that

$$
c_{2} \widetilde{c}_{3}=\frac{1+\mathcal{O}(h)}{1+\mathcal{O}(h)} \frac{1+\mathcal{O}(h)}{1+\mathcal{O}(h)}=1+\mathcal{O}(h) \quad(h \rightarrow 0)
$$

from (3.4). This holds in both cases $A(\alpha)= \pm A(\beta)$.

To calculate the Wronskian $\mathcal{W}\left(\boldsymbol{u}_{1}, \boldsymbol{u}_{2}\right)$, we recall that there exists a branch cut between $x_{1}$ and $x_{2}$. For this, we have to represent $\boldsymbol{u}_{1}$ or $\boldsymbol{u}_{2}$ by different branches. Let $\hat{x}$ denote a point obtained by rotating $x$ by the angle of $-2 \pi$ around $\alpha$, that is,

$$
\hat{x}-\alpha=e^{-2 \pi i}(x-\alpha) .
$$

Then, we rewrite $\boldsymbol{u}_{2}$ in terms of $\hat{x}$. When $A(\alpha)=\lambda$,

$$
\sqrt{A(x)-\lambda}=\sqrt{e^{2 \pi i}(A(\hat{x})-\lambda)}=-\sqrt{(A(\hat{x})-\lambda)} .
$$

On the other hand, when $A(\alpha)=-\lambda$,

$$
\sqrt{A(x)+\lambda}=\sqrt{e^{2 \pi i}(A(\hat{x})+\lambda)}=-\sqrt{(A(\hat{x})+\lambda)} .
$$

Therefore, there is a sign change

$$
+z(x ; \alpha)=-z(\hat{x} ; \alpha)
$$

in both cases $A(\alpha)= \pm \lambda$. Since the sign of $z(x)$ changes and $c(x)=c(\hat{x})$, we find from the recurrence equation (2.3) that

$$
\boldsymbol{w}^{+}\left(x, h ; x_{2}\right)=\boldsymbol{w}^{-}\left(\hat{x}, h ; \hat{x_{2}}\right) .
$$

The representation of the function $H(x)$ is different in the cases where $A(\alpha)=\lambda$ or $A(\alpha)=-\lambda$. When $A(\alpha)=\lambda$,

$$
H(x)=\left(\frac{A(x)+\lambda}{A(x)-\lambda}\right)^{\frac{1}{4}}=\left(\frac{1}{e^{2 \pi i}} \frac{A(\hat{x})+\lambda}{A(\hat{x})-\lambda}\right)^{\frac{1}{4}}=e^{-\frac{\pi i}{2}}\left(\frac{A(\hat{x})+\lambda}{A(\hat{x})-\lambda}\right)^{\frac{1}{4}} .
$$


By contrast, when $A(\alpha)=-\lambda$,

$$
H(x)=\left(\frac{A(x)+\lambda}{A(x)-\lambda}\right)^{\frac{1}{4}}=\left(e^{2 \pi i} \frac{A(\hat{x})+\lambda}{A(\hat{x})-\lambda}\right)^{\frac{1}{4}}=e^{\frac{\pi i}{2}}\left(\frac{A(\hat{x})+\lambda}{A(\hat{x})-\lambda}\right)^{\frac{1}{4}} .
$$

That is, $H(x)=\mp i H(\hat{x})$ holds with $A(\alpha)= \pm \lambda$. In addition, this leads to

$$
Q(x)= \pm i Q(\hat{x})\left(\begin{array}{ll}
0 & 1 \\
1 & 0
\end{array}\right) .
$$

From (3.5), (3.6), and (3.7), we can rewrite $\boldsymbol{u}_{2}$ as

$$
\boldsymbol{u}_{2}= \pm i \boldsymbol{u}^{-}\left(\hat{x}, h ; \alpha, \hat{x_{2}}\right),
$$

and obtain

$$
\begin{aligned}
\mathcal{W}\left(\boldsymbol{u}_{1}, \boldsymbol{u}_{2}\right) & =\mathcal{W}\left(\boldsymbol{u}^{+}\left(x, h ; \alpha, x_{1}\right), \pm i \boldsymbol{u}^{-}\left(\hat{x}, h ; \alpha, \hat{x_{2}}\right)\right) \\
& =\mp 4 i \operatorname{det} Q w_{\text {even }}^{+}\left(\hat{x_{2}}, h ; x_{1}\right) .
\end{aligned}
$$

in each case $A(\alpha)= \pm \lambda$.

In the same way, we represent $\widetilde{\boldsymbol{u}_{3}}$ by the other branch to calculate $\mathcal{W}\left(\boldsymbol{u}_{4}, \widetilde{\boldsymbol{u}}_{3}\right)$. Let $\widetilde{x}$ denote a point obtained by rotating $x$ by the angle of $-2 \pi$ around $\beta$. When $A(\beta)= \pm \lambda, \widetilde{\boldsymbol{u}_{3}}$ is rewritten as

$$
\widetilde{\boldsymbol{u}_{3}}= \pm i \boldsymbol{u}^{+}\left(\widetilde{x}, h ; \beta, \widetilde{x_{3}}\right) \text {. }
$$

Therefore, $\mathcal{W}\left(\boldsymbol{u}_{4}, \widetilde{\boldsymbol{u}}_{3}\right)$ is calculated as

$$
\begin{aligned}
\mathcal{W}\left(\boldsymbol{u}_{4}, \widetilde{\boldsymbol{u}}_{3}\right) & =-\mathcal{W}\left( \pm i \boldsymbol{u}^{+}\left(x, h ; \beta, \widetilde{x_{3}}\right), \boldsymbol{u}^{-}\left(x, h ; \beta, x_{4}\right)\right) \\
& = \pm 4 i \operatorname{det} Q w_{\text {even }}^{+}\left(x_{4}, h ; \widetilde{x_{3}}\right) .
\end{aligned}
$$

We can find $\Gamma\left(x_{1}, \hat{x_{2}}\right)$ and $\Gamma\left(\widetilde{x_{3}}, x_{4}\right)$ along which $\operatorname{Re} z(x, \cdot)$ strictly increasing, and obtain

$$
w_{\text {even }}^{+}\left(\hat{x_{2}}, h ; x_{1}\right)=1+\mathcal{O}(h), \quad w_{\text {even }}^{+}\left(x_{4}, h ; \widetilde{x_{3}}\right)=1+\mathcal{O}(h)
$$

as $h \rightarrow 0$.

As a result, we obtain that when $A(\alpha) A(\beta)>0$,

$$
c_{3} \widetilde{c_{2}}=i^{2} \frac{w_{\text {even }}^{+}\left(\hat{x_{2}}, h ; x_{1}\right)}{w_{\text {even }}^{+}\left(x_{3}, h ; x_{2}\right)} \frac{w_{\text {even }}^{+}\left(x_{4}, h ; \widetilde{x_{3}}\right)}{w_{\text {even }}^{+}\left(x_{3}, h ; x_{2}\right)}=-1+\mathcal{O}(h)
$$

as $h \rightarrow 0$. In the case $A(\alpha) A(\beta)<0$,

$$
c_{3} \widetilde{c_{2}}=-i^{2} \frac{w_{\text {even }}^{+}\left(\hat{x_{2}}, h ; x_{1}\right)}{w_{\text {even }}^{+}\left(x_{3}, h ; x_{2}\right)} \frac{w_{\text {even }}^{+}\left(x_{4}, h ; \widetilde{x_{3}}\right)}{w_{\text {even }}^{+}\left(x_{3}, h ; x_{2}\right)}=1+\mathcal{O}(h)
$$

as $h \rightarrow 0$.

Here we return to equation (1.1). The spectral parameter $\lambda$ near $\lambda_{0}$ is an eigenvalue of $L$ if and only if $\boldsymbol{u}_{1}$ and $\boldsymbol{u}_{4}$ are linearly dependent, since $\boldsymbol{u}_{1} \in L^{2}\left(\mathbb{R}_{-}\right)$and $\boldsymbol{u}_{4} \in L^{2}\left(\mathbb{R}_{+}\right)$. That is, we consider the condition

$$
\mathcal{W}\left(\boldsymbol{u}_{1}, \boldsymbol{u}_{4}\right)=0 .
$$


From (3.2) and (3.3), we know that the Wronskian $\mathcal{W}\left(\boldsymbol{u}_{1}, \boldsymbol{u}_{4}\right)$ is expressed in terms of $\boldsymbol{u}_{j}$ and $\widetilde{\boldsymbol{u}_{k}}(j, k \in\{2,3\})$ as

$$
\mathcal{W}\left(\boldsymbol{u}_{1}, \boldsymbol{u}_{4}\right)=c_{2} \widetilde{c}_{3} \mathcal{W}\left(\boldsymbol{u}_{2}, \widetilde{\boldsymbol{u}}_{3}\right)-\widetilde{c}_{2} c_{3} \mathcal{W}\left(\widetilde{\boldsymbol{u}}_{2}, \boldsymbol{u}_{3}\right) .
$$

Since $\boldsymbol{u}_{j}$ and $\widetilde{\boldsymbol{u}_{j}}$ are linearly dependent and satisfy

$$
\widetilde{\boldsymbol{u}}_{2}=e^{-i I(\lambda) / h} \boldsymbol{u}_{2}, \widetilde{\boldsymbol{u}}_{3}=e^{i I(\lambda) / h} \boldsymbol{u}_{3},
$$

condition (3.8) is equivalent to

$$
c_{2} \widetilde{c}_{3} e^{i I(\lambda) / h}-\widetilde{c}_{2} c_{3} e^{-i I(\lambda) / h}=0 .
$$

That is, $I(\lambda)$ satisfies

$$
I(\lambda)+\frac{h}{2 i} \log \left(-\frac{c_{2} \widetilde{c}_{3}}{\widetilde{c}_{2} c_{3}}\right)=\left(k+\frac{1}{2}\right) \pi h
$$

for some integer $k$.

From Lemma 3.1, when $A(\alpha) A(\beta)>0$,

$$
\log \left(-\frac{c_{2} \widetilde{c}_{3}}{\widetilde{c}_{2} c_{3}}\right)=\log (1+\mathcal{O}(h))=\mathcal{O}(h) \quad(h \rightarrow 0) .
$$

On the other hand, when $A(\alpha) A(\beta)<0$,

$$
\log \left(-\frac{c_{2} \widetilde{c}_{3}}{\widetilde{c}_{2} c_{3}}\right)=\log (-1+\mathcal{O}(h))=\pi i+\mathcal{O}(h) \quad(h \rightarrow 0) .
$$

In conclusion, the quantization condition for eigenvalues $\lambda$ is given by

$$
I(\lambda)=\left(k+\frac{1}{2}\right) \pi h+\mathcal{O}\left(h^{2}\right) \quad(h \rightarrow 0),
$$

in the case $A(\alpha) A(\beta)>0$. Similarly, we obtain

$$
I(\lambda)=k \pi h+\mathcal{O}\left(h^{2}\right) \quad(h \rightarrow 0),
$$

in the case $A(\alpha) A(\beta)<0$.

\section{Eigenvalue PRoblem for the NON-SELF-ADJoint CASE}

In this section, we consider the eigenvalue problem:

$$
L_{\varepsilon} \boldsymbol{u}(x)=\lambda \boldsymbol{u}(x), \quad L_{\varepsilon}:=\left(\begin{array}{cc}
i h \frac{d}{d x} & -i A_{\varepsilon}(x) \\
i A_{\varepsilon}(x) & -i h \frac{d}{d x}
\end{array}\right),
$$

for $A_{\varepsilon}(x)=A(x)+i \varepsilon B(x)$ with $\varepsilon>0$. First we consider the quantization condition for the eigenvalues. Here we assume that $A(x)$ satisfies Assumption (A1) and $B(x)$ is real-valued, analytic and bounded on $\mathbb{R}$.

Let $D\left(\lambda_{0}, \varepsilon_{0}\right)=\left\{x \in \mathbb{C} ;\left|x-\lambda_{0}\right|<\varepsilon_{0}\right\}$ for a positive $\varepsilon_{0}$. Under Assumption (A1), for all $\lambda \in D\left(\lambda_{0}, \varepsilon_{0}\right)$ and $\varepsilon \in\left(0, \varepsilon_{0}\right]$, there exist zeros of $A_{\varepsilon}(x)^{2}-\lambda^{2}, \alpha(\lambda, \varepsilon)$ and $\beta(\lambda, \varepsilon)$ such that $\alpha\left(\lambda_{0}, 0\right)=\alpha_{0}$ and $\beta\left(\lambda_{0}, 0\right)=\beta_{0}$. We simply write them as $\alpha_{\varepsilon}$ and $\beta_{\varepsilon}$, and define the action integral $I(\lambda, \varepsilon)$ :

$$
I(\lambda, \varepsilon)=\int_{\alpha_{\varepsilon}(\lambda)}^{\beta_{\varepsilon}(\lambda)} \sqrt{A_{\varepsilon}(t)^{2}-\lambda^{2}} d t .
$$


In addition, the exact WKB solutions for (4.1) are given by replacing $A(x)$ with $A_{\varepsilon}(x)$ in (2.1), and we denote those solutions by $\boldsymbol{u}^{ \pm}\left(x, h, \varepsilon ; \gamma, x_{0}\right)$.

We choose $\alpha_{\varepsilon}$ and $\beta_{\varepsilon}$ for the base points of the phase function $z(x, \varepsilon)$. The Stokes lines which pass through the points $\alpha_{\varepsilon}$ and $\beta_{\varepsilon}$ are drown in Figure 4.

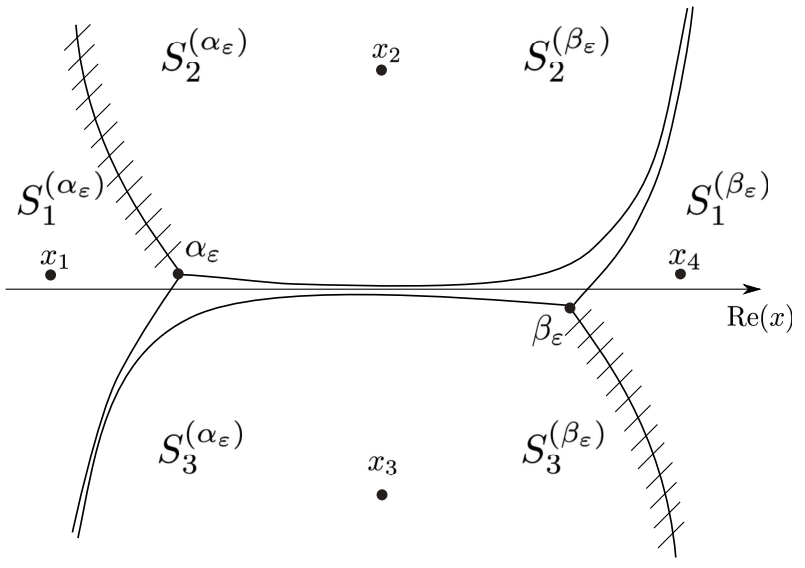

Figure 4. The Stokes lines for a sufficiently small $\varepsilon . S_{j}^{\left(\alpha_{\varepsilon}\right)}$ and $S_{k}^{\left(\beta_{\varepsilon}\right)}$ indicate the sector which is generated by the Stokes lines emanating from $\alpha_{\varepsilon}$ and $\beta_{\varepsilon}$ respectively.

The Stokes lines continuously change with respect to $\varepsilon$ from the case of $\varepsilon=0$, since $\alpha_{\varepsilon}, \beta_{\varepsilon}$ and $z(x, \varepsilon)$ are continuous with respect to $\varepsilon$. Here, we assume that $\varepsilon$ is sufficiently small, and take base points as in Figure 4 . Then, we can derive the quantization conditions for eigenvalues of $L_{\varepsilon}$ in the same way as the previous section.

Lemma 4.1. Assume (A1), and let $B(x)$ be real-valued, analytic and bounded on $\mathbb{R}$. In the case $A\left(\alpha_{0}\right)=A\left(\beta_{0}\right)$, there exist positive constants $\varepsilon_{0}$ and $h_{0}$, and a function $r_{+}(\lambda, \varepsilon, h)$ bounded on $D\left(\lambda_{0}, \varepsilon_{0}\right) \times\left(0, \varepsilon_{0}\right] \times\left(0, h_{0}\right]$ such that $\lambda \in D\left(\lambda_{0}, \varepsilon_{0}\right)$ is an eigenvalue of $L_{\varepsilon}$ for $\varepsilon \in\left(0, \varepsilon_{0}\right]$ and $h \in\left(0, h_{0}\right]$ if and only if

$$
I(\lambda, \varepsilon)=\left(k+\frac{1}{2}\right) \pi h+h^{2} r_{+}(\lambda, \varepsilon, h)
$$

holds for some integer $k$. In the case $A\left(\alpha_{0}\right)=-A\left(\beta_{0}\right)$, there exist positive constants $\varepsilon_{0}$ and $h_{0}$, and a function $r_{-}(\lambda, \varepsilon, h)$ bounded on $D\left(\lambda_{0}, \varepsilon_{0}\right) \times$ $\left(0, \varepsilon_{0}\right] \times\left(0, h_{0}\right]$ such that $\lambda \in D\left(\lambda_{0}, \varepsilon_{0}\right)$ is an eigenvalue of $L_{\varepsilon}$ for $\varepsilon \in\left(0, \varepsilon_{0}\right]$ and $h \in\left(0, h_{0}\right]$ if and only if

$$
I(\lambda, \varepsilon)=k \pi h+h^{2} r(\lambda, \varepsilon, h)
$$

holds for some integer $k$.

Now, we assume Assumption (A2) for $A_{\varepsilon}(x)$, which results in a symmetry of the action integral $I(\lambda, \varepsilon)$ and the exact WKB solutions $\boldsymbol{u}^{ \pm}\left(x, h, \varepsilon ; \gamma, x_{0}\right)$ with respect to complex conjugation. 
Lemma 4.2. Under Assumption (A2), the action integral $I(\lambda, \varepsilon)$ is equal to the complex conjugate of $I(\bar{\lambda}, \varepsilon)$ :

$$
\overline{I(\bar{\lambda}, \varepsilon)}=I(\lambda, \varepsilon) \text {. }
$$

Proof. By a simple calculation, we find that $-\overline{\beta_{\varepsilon}}$ and $-\overline{\alpha_{\varepsilon}}$ are zeros of $A_{\varepsilon}(x)^{2}-\bar{\lambda}^{2}$ under Assumption (A2). That is, $I(\bar{\lambda}, \varepsilon)$ is represented as

$$
I(\bar{\lambda}, \varepsilon)=\int_{-\overline{\beta_{\varepsilon}}}^{-\overline{\alpha_{\varepsilon}}} \sqrt{\bar{\lambda}^{2}-A_{\varepsilon}(t)^{2}} d t .
$$

We take the complex conjugate of this, and obtain that

$$
\overline{I(\bar{\lambda}, \varepsilon)}=\int_{-\bar{\beta}_{\varepsilon}}^{-\overline{\alpha_{\varepsilon}}} \sqrt{\overline{\left(\bar{\lambda}^{2}-A_{\varepsilon}(t)^{2}\right)}} d \bar{t}=\int_{-\bar{\beta}_{\varepsilon}}^{-\overline{\alpha_{\varepsilon}}} \sqrt{\lambda^{2}-{\overline{A_{\varepsilon}(t)}}^{2}} d \bar{t} .
$$

Then, we change the variable from $t$ to $-\bar{t}$,

$$
\overline{I(\bar{\lambda}, \varepsilon)}=-\int_{\beta_{\varepsilon}}^{\alpha_{\varepsilon}} \sqrt{\lambda^{2}-{\overline{A_{\varepsilon}(-\bar{t}}}^{2}} d t=\int_{\alpha_{\varepsilon}}^{\beta_{\varepsilon}} \sqrt{\lambda^{2}-A_{\varepsilon}(t)^{2}} d t
$$

This is just the action integral.

We denote the exact WKB solutions for the equation

$$
L_{\varepsilon} \boldsymbol{v}(x)=\bar{\lambda} \boldsymbol{v}(x)
$$

by $\boldsymbol{v}^{ \pm}$. Then, $\boldsymbol{v}^{ \pm}$is obtained by replacing $\lambda$ with $\bar{\lambda}$. Under Assumtion (A2), $\boldsymbol{u}^{ \pm}$and $\boldsymbol{v}^{ \pm}$also have the following symmetry relations.

Lemma 4.3. Under Assumption (A2), if $\overline{A_{\varepsilon}(-\bar{x})}=A_{\varepsilon}(x)$, the exact WKB solutions $\boldsymbol{u}^{ \pm}\left(x, h, \varepsilon ; \gamma, x_{0}\right)$ and $\boldsymbol{v}^{ \pm}\left(x, h, \varepsilon ; \gamma, x_{0}\right)$ satisfy

$$
\boldsymbol{u}^{ \pm}\left(x, h, \varepsilon ; \gamma, x_{0}\right)=\left(\begin{array}{cc}
1 & 0 \\
0 & -1
\end{array}\right) \overline{\boldsymbol{v}^{\mp}\left(-\bar{x}, h, \varepsilon ;-\bar{\gamma},-\overline{x_{0}}\right)}
$$

and if $\overline{A_{\varepsilon}(-\bar{x})}=-A_{\varepsilon}(x)$, then

$$
\boldsymbol{u}^{ \pm}\left(x, h, \varepsilon ; \gamma, x_{0}\right)= \pm\left(\begin{array}{cc}
0 & -1 \\
1 & 0
\end{array}\right) \overline{\boldsymbol{v}^{\mp}\left(-\bar{x}, h, \varepsilon ;-\bar{\gamma},-\overline{x_{0}}\right)} .
$$

Proof. Let $\overline{A_{\varepsilon}(-\bar{x})}=A_{\varepsilon}(x)$. By taking the complex conjugate and changing the variable $x$ to $-\bar{x}$ for the functions $z$ and $\boldsymbol{w}^{ \pm}$of the solutions $\boldsymbol{v}^{ \pm}$, we obtain

$$
\overline{z(-\bar{x}, \varepsilon ;-\bar{\gamma} ; \bar{\lambda})}=-z(x, \varepsilon ; \gamma ; \lambda)
$$

and

$$
\overline{\boldsymbol{w}^{ \pm}\left(-\bar{x}, h, \varepsilon ;-\overline{x_{0}} ; \bar{\lambda}\right)}=\boldsymbol{w}^{\mp}\left(x, h, \varepsilon ; x_{0} ; \lambda\right) .
$$

In the same way, for the matrix function $Q(x, \varepsilon)$ of $\boldsymbol{v}^{ \pm}$,

$$
\overline{Q(-\bar{x}, \varepsilon ; \bar{\lambda})}=Q(x, \varepsilon ; \lambda)\left(\begin{array}{ll}
0 & 1 \\
1 & 0
\end{array}\right) .
$$


Here, we recall that the solutions $\boldsymbol{v}^{ \pm}$is of the form

$$
\boldsymbol{v}^{ \pm}\left(x, h, \varepsilon ; \gamma, x_{0}\right)=\left(\begin{array}{cc}
1 & 1 \\
-i & i
\end{array}\right) e^{ \pm z(x, \varepsilon ; \gamma) / h} Q(x, \varepsilon)\left(\begin{array}{ll}
0 & 1 \\
1 & 0
\end{array}\right)^{\frac{1 \pm 1}{2}} \boldsymbol{w}^{ \pm}\left(x, h, \varepsilon ; x_{0}\right) .
$$

By taking the complex conjugate and changing the variable $x$ to $-\bar{x}$, and using above, we obtain the first relation

$$
\left(\begin{array}{cc}
1 & 0 \\
0 & -1
\end{array}\right) \overline{\boldsymbol{v}^{\mp}\left(-\bar{x}, h, \varepsilon ;-\bar{\gamma},-\overline{x_{0}}\right)}=\boldsymbol{u}^{ \pm}\left(x, h, \varepsilon ; \gamma, x_{0}\right) .
$$

If $\overline{A_{\varepsilon}(-\bar{x})}=-A_{\varepsilon}(x)$, then we find that

$$
\begin{aligned}
\overline{z(-\bar{x}, \varepsilon ;-\bar{\gamma} ; \bar{\lambda})} & =-z(x, \varepsilon ; \gamma ; \lambda), \\
\overline{\boldsymbol{w}^{ \pm}\left(-\bar{x}, h, \varepsilon ;-\overline{x_{0}} ; \bar{\lambda}\right)} & =\left(\begin{array}{cc}
1 & 0 \\
0 & -1
\end{array}\right) \boldsymbol{w}^{\mp}\left(x, h, \varepsilon ; x_{0} ; \lambda\right),
\end{aligned}
$$

and

$$
\overline{Q(-\bar{x}, \varepsilon ; \bar{\lambda})}=-i\left(\begin{array}{cc}
0 & 1 \\
-1 & 0
\end{array}\right) Q(x, \varepsilon ; \lambda)\left(\begin{array}{cc}
0 & 1 \\
-1 & 0
\end{array}\right) .
$$

From this property, the second relation also follows.

Here we take the base points $x_{1} \in S_{1}^{\left(\alpha_{\varepsilon}\right)}$ and $x_{2} \in S_{2}^{\left(\alpha_{\varepsilon}\right)}$ so that $-\overline{x_{1}} \in S_{1}^{\left(\beta_{\varepsilon}\right)}$ and $-\overline{x_{2}} \in S_{3}^{\left(\beta_{\varepsilon}\right)}$, and set the exact WKB solutions for (4.1):

$$
\begin{cases}\boldsymbol{u}_{1}=\boldsymbol{u}^{+}\left(x, h, \varepsilon ; \alpha_{\varepsilon}, x_{1}\right), & \boldsymbol{u}_{2}=\boldsymbol{u}^{+}\left(x, h, \varepsilon ; \alpha_{\varepsilon}, x_{2}\right) \\ \boldsymbol{u}_{3}=\boldsymbol{u}^{-}\left(x, h, \varepsilon ; \alpha_{\varepsilon},-\overline{x_{2}}\right) & \boldsymbol{u}_{4}=\boldsymbol{u}^{-}\left(x, h, \varepsilon ; \beta_{\varepsilon},-\overline{x_{1}}\right) .\end{cases}
$$

In addition, let us define a function $W(\lambda, \varepsilon)$ by the Wronskian of $\boldsymbol{u}_{1}$ and $\boldsymbol{u}_{4}$, that is,

$$
W(\lambda, \varepsilon):=\mathcal{W}\left(\boldsymbol{u}_{1}, \boldsymbol{u}_{4}\right) .
$$

We also take the solutions for (4.5) as

$$
\left\{\begin{array}{l}
\boldsymbol{v}_{1}=\boldsymbol{v}^{+}\left(x, h, \varepsilon ;-\overline{\beta_{\varepsilon}}, x_{1}\right), \quad \boldsymbol{v}_{2}=\boldsymbol{v}^{+}\left(x, h, \varepsilon ;-\overline{\alpha_{\varepsilon}}, x_{2}\right) \\
\boldsymbol{v}_{3}=\boldsymbol{v}^{-}\left(x, h, \varepsilon ;-\overline{\alpha_{\varepsilon}},-\overline{x_{2}}\right) \quad \boldsymbol{v}_{4}=\boldsymbol{v}^{-}\left(x, h, \varepsilon ;-\overline{\alpha_{\varepsilon}},-\overline{x_{1}}\right) .
\end{array}\right.
$$

Then, we see that

$$
W(\lambda, \varepsilon)= \pm \overline{W(\bar{\lambda}, \varepsilon)}
$$

by the definition of $W(\lambda, \varepsilon)$ and applying Lemma 4.3. Here, the sign of (4.6) is dependent on whether $A_{\varepsilon}(x)=\overline{A(-\bar{x})}$ or $A_{\varepsilon}(x)=-\overline{A(-\bar{x})}$.

Now, we recall that $W(\lambda, \varepsilon)$ is represented as

$$
W(\lambda, \varepsilon)=a(\lambda, \varepsilon, h) e^{i I(\lambda, \varepsilon) / h}+b(\lambda, \varepsilon, h) e^{-i I(\lambda, \varepsilon) / h},
$$

where $a$ and $b$ are some functions with $a=1+\mathcal{O}(h)$ and $b=1+\mathcal{O}(h)$ or $-1+\mathcal{O}(h)$ as $h \rightarrow 0$. In particular, $a$ and $b$ satisfy

$$
a(\lambda, \varepsilon, h)= \pm \overline{b(\bar{\lambda}, \varepsilon, h)}
$$


since $W(\lambda, \varepsilon)$ satisfy (4.6), and $I(\lambda, \varepsilon)$ also satisfy Lemma 4.2. Moreover, (4.7) is rewritten as

$$
W(\lambda, \varepsilon)=b(\lambda, \varepsilon, h) e^{-I(\lambda, \varepsilon) / h}\left(\exp \left(\frac{2 i}{h}\left(I(\lambda, \varepsilon)+h^{2} r(\lambda, \varepsilon, h)\right)\right)+1\right),
$$

where

$$
r(\lambda, \varepsilon, h)=\frac{1}{2 i h} \log \frac{a(\lambda, \varepsilon, h)}{b(\lambda, \varepsilon, h)} .
$$

Then, we use (4.8) and obtain that

$$
r(\lambda, \varepsilon, h)=\overline{r(\bar{\lambda}, \varepsilon, h)} .
$$

Here we take $I(\lambda, \varepsilon, h)$ as

$$
I(\lambda, \varepsilon, h):=I(\lambda, \varepsilon)+h^{2} r(\lambda, \varepsilon, h) .
$$

This is a function from a neighborhood of $\lambda_{0}$ to one of $I\left(\lambda_{0}, 0\right)$. In particular, $r(\lambda, \varepsilon, h)$ is holomorphic near $\lambda_{0}$, and $I(\lambda, \varepsilon)$ satisfies $\frac{d I}{d \lambda}\left(\lambda_{0}, 0\right) \neq 0$. This implies that $I(\lambda, \varepsilon, h)$ has an inverse function $I^{-1}(\zeta, \varepsilon, h)$, and the eigenvalues of $L_{\varepsilon}$ near $\lambda_{0}$ are given by

$$
\lambda_{\varepsilon, k}=I^{-1}\left(c_{k} \pi h, \varepsilon, h\right), \quad k \in \mathbb{Z},
$$

where $c_{k}=k$ or $k+1 / 2$. In addition, we know that $I(\lambda, \varepsilon, h)$ is real for $\lambda \in \mathbb{R}$, since $r(\lambda, \varepsilon, h)$ and $I(\lambda, \varepsilon)$ is real for $\lambda \in \mathbb{R}$ by (4.9) and Lemma 4.2 . That is, the eigenvalues near $\lambda_{0}$ are real.

\section{REFERENCES}

[1] C.M. Bender: Introduction to $\mathcal{P} \mathcal{T}$-symmetric Quantum Theory, Contemporary Physics 46(4), 277-292 (2005).

[2] C.M. Bender and S. Boettcher: Real Spectra in Non-Hermitian Hamiltonians Having PT Symmetry, Physical Review Letters 80(24), (1998).

[3] C.M. Bender and H.F. Jones: Wentzel-Kramers-Brillouin analysis of $\mathcal{P} \mathcal{T}$-Symmetric Sturm-Liouville problems, Physical Review A 85(5), (2012).

[4] N. Boussekkine and N. Mecherout: $\mathcal{P} \mathcal{T}$-symmetry and potentiel well. The simple well case, Mathematische Nachrichten 289(1), 13-27 (2016).

[5] S. Fujiié, C. Lasser, and L. Nédélec: Semiclassical resonances for a two-level Schrödinger operator with a conical intersection, Asymptotic Analysis 65(1-2), 17-58 (2009).

[6] S. Fujiié and J. Wittsten: Quantization conditions of eigenvalues for semiclassical Zakharov-Shabat systems on the circle, preprint (2017), arXiv:1703.08352.

[7] C. Gérard and A. Grigis: Precise estimates of tunneling and eigenvalues near a potential barrier, Journal of differential equations 72(1), 149-177 (1988).

[8] N. Mecherout, N. Boussekkine, T. Ramond and J. Sjöstrand: $\mathcal{P} \mathcal{T}$-symmetry and Schrödinger operators. The double well case, Mathematische Nachrichten 289(7), 854-887 (2016).

[9] V.E. Zakharov and A.B. Shabat: Exact Theory of Two-dimensional Self-focusing and One-dimensional Self-modulation of Wave in Nonlinear Media, Journal of Experimental and Theoretical Physics 34(1), 62-69 (1972). 